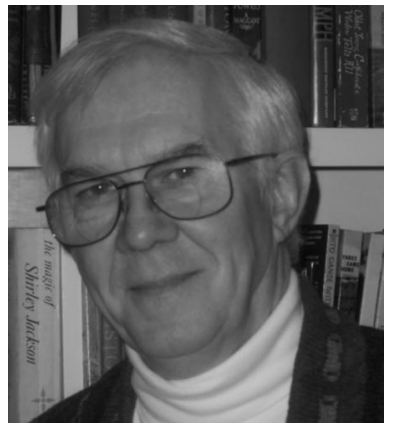

\section{Designing Optical Systems, Part II: Optimization}

Last month I analyzed some of the aberrations in current training of optical designers. I noted that much of the powerful software is purchased by technical persons (and their managers) who have little or no background in optics. They attack new problems not from a fundamental understanding of the system, but based on their last project. This situation should cause concern, for there are few curricula and courses beyond the strong curriculum at the Optical Sciences Center at the University of Arizona and some modest offerings at other institutions that do funded optics research.

Yet, the introduction of optical technology continues apace in our modern world. Now, the variety of detectors, beyond the eye and photographic film, is amazing. So whatever type of training in optical design that is to be done, it should extend beyond lens design to illumination engineering, diffractive optics, optomechanical design, and, perhaps, fabrication. When you look at the variety of optical systems that have been invented or perfected within the last decade, you realize that the last thing in the world we need is another lens that is nothing more than a reoptimized double Gauss. For those organizations that need trained persons in optical design, particularly when many of their employees are already in the middle of things, their training has to take place at a distance and on the fly.

Both SPIE and the optical design software houses offer short courses on various aspects of optical design. Many times, these courses permit a novice designer to get an answer to his or her current problem. However, the courses cannot, due to time limitations, teach the fundamentals on which many of the short courses are based. In addition, there are no homework problems, no tests, and no evaluation of student performance. Yet that is what is needed. For it would be better to mark a designer as incompetent by issuing an Unsatisfactory or an " $F$ " in a design course, than to have his or her expensive design perform badly after all the parts have been fabricated and assembled.

What are needed are distance learning environments wherein students practice through problem sets and demonstrate competence through tests. Perhaps the best current source of such training is the program at the Optical
Science Center at the University of Arizona. It offers a number of the core master's level courses online using a traditional semester course schedule with a weekly set of lectures. It represents one of the few sources of optics teaching that requires evidence of mastery of the material. Currently, the offerings are limited to about a half dozen courses each semester, according to the schedule posted on the web (http://www.eu.arizona.edu/dist/ opti_courses.html). These courses can be combined with a one-semester residency to earn a master's of science in optical science or a graduate certificate, if it is not possible to fulfill the residency requirement.

The program is certainly one of the most comprehensive sources of distance education in this critical field. There are topics such as optomechanical design, diffractive optics, lens design, and illumination design that are not covered in any detail. As it is, the program is constrained by technical resources to tape the lectures. I think it is fair to ask if the current offerings at Arizona are sufficient to provide enough variety and opportunities for training.

Is there a way to optimize our professional education in optical design and engineering? What are the constraints that must be imposed on any proposed solution to get a realistic answer? If you think I am now about to reveal "The Answer," I am sorry to disappoint you. These editorials are, I hope, part of an optimization process. Considering the import and commitments needed to arrive at an acceptable solution, it will take a lot more than a few sentences to design additional approaches to producing qualified optical designers.

I do have some ideas about approaches that might be used. For example, I think that any attempt to produce qualified optical designers must maintain an academic base. This is because any curriculum together with the requirements for student preparation must be determined by an academic body. There must be oversight of program performance and approval of any modifications to the curriculum. This is not the job of a business or a professional society. I don't think this academic base must necessarily be attached to a research institution. Instead, a solid academically certified college or university might be sufficient. I do think, as solid as the Optical Sciences Center program appears to be, there is a need for similar resources distributed globally with different technical emphases. This would permit others with expertise in 
complementary fields to contribute to the training in their specialties.

The instruction methods would almost certainly be web based. Arizona uses web streaming as one of several delivery methods, but right now course delivery is tied to a semester schedule. Considering that optical design today is a computer-based discipline, a real campus may not be needed, although a residency similar to Arizona's or a summer workshop with laboratories would be useful. Also, with the capability of video-based Internet chat (see my March 2004 editorial, "Grandparent Technology") face-to-face tutorial sessions become feasible.

Such a program might involve a professional society close to the optical design community, such as SPIE. While academic institutions might be able to establish and maintain standards and even provide certification (something that SPIE has pointedly avoided), they could not, with the few exceptions, connect to the optics community and identify the resources that would be needed to establish a strong program. The optics community should identify the current and future needs for training in optical design, provide information to make others aware of these needs, and formulate a program to meet them.

Donald C. O'Shea Editor 